\title{
PNEUMATIC SYSTEMS FOR AUTOMATIC SORTING EQUIPMENT
}

\author{
Iveta Onderováa, ${ }^{a}, V_{\text {iliam ČaČKo }}^{a}$, L’udovít Kolláth $^{a}$, L’ubomír Šooš $^{a}$, \\ Andrej Smelík ${ }^{b}$, Alexander Varga ${ }^{b}$ \\ a Slovak University of Technology in Bratislava, Faculty of Mechanical Engineering, Institute of Manufacturing \\ Systems, Environmental Technology and Quality Management, Námestie Slobody 17, 81231 Bratislava, Slovak \\ Republic \\ ${ }^{b}$ MicroStep, spol. s r.o., Vajnorská 158, 83104 Bratislava, Slovak Republic \\ * corresponding author: iveta.onderova@stuba.sk
}

\begin{abstract}
The aim of the research work was focused on a research of manipulators design using suction grippers. The results of the research can be applied in the design of jigs - manipulators for handling thin sheets. The FME analysis was used in the investigation to show the course of elastic deformations and stresses in stressed areas. An experimental observation of the change in the free deflection of the workpiece from the positioning of the suction cups under gravity load by means of a laser 3D scanner was carried out for the FEM deflection detection method.
\end{abstract}

KEYWORDS: Effector, handling metal sheets, suction gripper, 3D scanner, object handling.

\section{INTRODUCTION}

In the solution, a modular system has been designed for the automatic loading of a material to the working area of the cutting heads, a system of automatic gripping, shifting and filing of the material in the cutting process and sorting it after the cutting process. The modular system is designed for cutting sheets and for the division of open and closed profiles of different cross sections. Moving and pivoting modules in the work zone are interpolated moving axes of the CNC system. These modules are equipped with automatically operated sensing grip units for an accurate positioning of the material and for an accurate identification of its dimensions.

The goal was to create automated cells for fully automated cutting workstations with a material flow customizable to customer's requirements. Special robotic kinematics are incorporated into the cutting system to extend the cutting capabilities for more complicated spatial formations controlled directly from the CNC machine control system as well as to manipulate and sort the finished parts. [1-3]

In addition to the development of the control system for the individual modules of these nodes, the development of an information system for the production management of such a node or group of nodes creating automatic cutting workplaces was carried out. Such a subsystem can easily be connected to the enterprise information system. In the system, apart from input operations for dividing the parts into individual blanks, a comprehensive database is kept containing information about the subsequent operations necessary to make the final products, bending, assembly, dyeing, the individual operations being defined by their capacity, and the manner and capacity for moving the blanks there. It is possible to include the transfer of semi-products on pallets, handling devices or conveyors into the system. This system allows an automatic sorting of products at the cutting centre's exit as the products are further processed or dispatched, which increases the efficiency of the entire production process. The user always has the information on the current state of the production and the position of individual parts in the production. This system also allows for more efficient planning of the cutting process with regard to the capacity of the workplaces carrying out subsequent operations as well as the capacity of handling operations. [4, 5]

The application area of such complex cutting nodes is characterized by the need for a great deal of cutting, such as the production of ships, railway rolling stock, steel structures of production halls, bridges and, wind power plant turbines. In the world, a demand is growing for them and it is related to the effort to increase the degree of automation of production.

The article presents the results of a project focused on the research of state-of-the-art technical solutions in the area of CNC machines for the cutting of materials by energy technologies - plasma, oxygen, highpressure water and laser. The project focuses on the development of nodes allowing a modular construction of complex cutting centres with a combination of splitting technologies, additional drilling, thread cutting and labelling technologies, with the possibility of automatic loading of semi-finished products and automatic unloading of finished parts. The solution also includes the automatic removal of waste emerging from the cutting process. [5] 7 ] 


\section{Automatic SySTEM FOR LASER CUTTING MACHINES}

The aim of the project was to create a modular automatic handling system for the productive operation of MSF laser cutting machines.

In cooperation with STU at MicroStep, the workplace of the researcher, the final stage of the design of the loading and sorting system for laser cutting machines was carried out in the third stage of the solution (2017), as well as the development of the software for these types of complexes. The proposed modular system under the name MSLoad is described in the next section. In the next stage of the solution, the solution will be fine-tuned according to the experience gained from the functional models. The assembly completes the section for automatic sorting of cut out parts. The automatic sorting system is labelled MSSort. In the third stage, work on the creation of the software for the management of this type of complex was carried out in parallel.

\subsection{MS-SorT}

The MSLoad automatic loading system can be supplemented with an automatic sorting system as shown in Fig. 1. The individual parts of the system are marked as follows (following the previous numbering):

- 10. Pallets, containers for sorted parts

- 11. Sorting -- the sorting position of the grate of the cutting machine

- 12. Portal of the sorting section

- 13. Sorting - sorting support with gripper head

There is a gantry system 12 with one or two working supports 13 which are fitted with grippers. Below this gantry system, there is a place 11 where the working rack of the MSF cutting machine extends. In Fig. 1 there is a version of MSLoad with a tower stack. The system can also work in a tower-free assembly. In the sorting section, the individual parts are gripped from the cut material and deposited on the pallet-container 10. Removal of the remainder of the material after the parts are removed is performed by the ridge bolt after returning the sorted pallet into the laser exchange table. This amount is moved on a pallet located at the top of the loading trolley. Simultaneously, the bottom of the loading trolley is loaded with a new blank. with loading a new blank as well as described above.

The MSLoad + MSSort + MSF assembly works with three working palettes (grids) that run in the cutting system. At this point, one pallet in the cutting machine can be cut, the second pallet is on the exchange table (the remainder of the sheet is cut into a new blank) and the third pallet is at the position of the shredder). Considering the exchange table has two positions, the top and the bottom, then three jobs are available for three pallets. If the system works without sorting and the cut blank is moved along with the remainder on the pallet, then the system works with two pallets. If the system is working with sorting, then the pallet always comes out of the cutting machine to the upper position of the exchange table and a pallet from the bottom position of the exchange table enters the cutting machine. The palette from position 11 is always entered into the lower position of the MSF exchange table and the palette from the top position of the exchange table goes into the sorting machine. The remainder after the sorting is moved from the bottom position of the exchange table and a new blank is then loaded into it.

The grip head is designed to be adapted to the particular class of parts to be grasped. In addition to moving in the XYZ rectangle, the head also shoots. A universal solution for this node cannot exist, and therefore, in the MSSort system, the possibility of an automatic exchange of grip units is considered.

The grip units are automatically changed (dimension change or whole unit replacement), depending on which part is to be unloaded. The CAM cutting-out system for the entire automated complex generates, in addition to the cutting plan itself, a splitting plan that will also include the information about the type of the gripper, its parameters (the head itself can be automatically configured) and its orientation, The heads must be designed for a specific class of parts for a particular customer. The gripper cartridge may be located beneath the parking part of the grading portal.

\subsection{GRAB handLe of the SORTING-SORTING SYSTEM}

The problems of the resulting sheet metal deflection are clearly mathematical tasks. However, they require complex mathematical operations. If we had to make these calculations manually without using computational and simulation programs, that would cost us not only time but also there would be a lot of errors. By doing so, the error in each calculation is transferred to the next, so the whole simulation becomes inaccurate. Therefore, in the first step, the kinematic model of the board was correctly determined. 8, 9]

In the ANSYS program, a metal sheet of $1000 \times$ $900 \mathrm{~mm}$ was modelled. The output of this program was a macro in which a four-pronged calculation element was defined based on the kinematic model of the board, the coordinates of the gripping elements, which can be varied according to the position of the suction cups and the created mathematical mask. After entering exact coordinates, ANSYS has created three files that further loaded MATLAB, as there is no possibility to directly enter FEM parameters such as the calculation element in this program.

In the next step, a SolidWorks board model was created, where we directly followed the simulation of the folds. In this program, we had to create a model for each type of the gripping element and for each material thickness. Three material thicknesses 


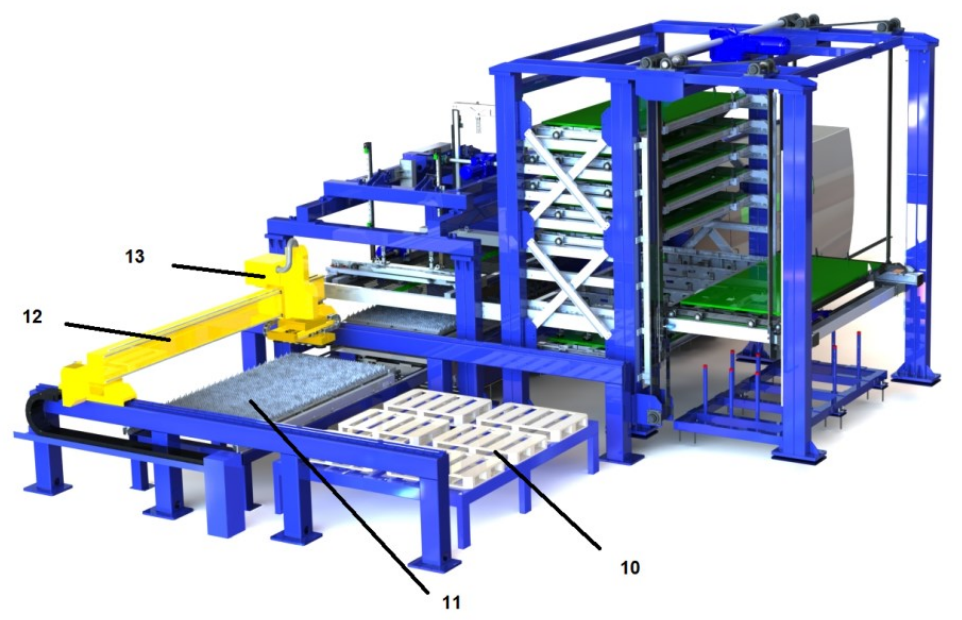

FiguRE 1. MSLoad + MSSort + MSF - view from the sorting system.
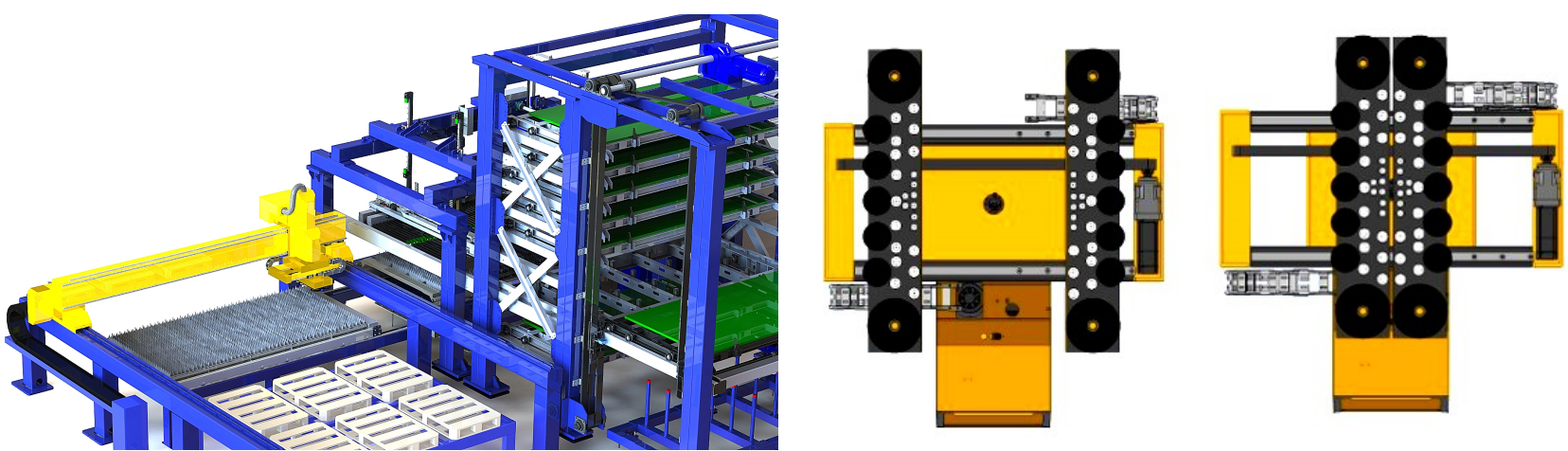

FiguRE 2. Grab handle of the sorting - sorting system.

of $0.67 \mathrm{~mm}$ were used, which are used for interior visual elements and $0.97 \mathrm{~mm}, 1.6 \mathrm{~mm}$ thickness, which are used for bodywork and car safety features. This material thickness input option has been programmed in MATLAB.

In the experimental part, a simple gripping head made of aluminium profiles was created. The effector and suction cups were used byused were made by FESTO. The head was composed of a main structure, on which four shoulders were attached at the ends of which were placed the suction cups. These arms were fully manipulated manipulatable in all directions, so different layouts could be arranged across the entire surface of the manipulated sheet. In the next step, this head was attached to the SEF VRS1D handling robot and Leica's Laser Tracker and T-Scane values were measured for the resulting sheet metal real deflections. [10, 11 .

\subsection{EXPERIMENT OF THE DISTRIBUTION OF THE SUCTION CUPS ON THE SORTING HEAD}

The purpose of the experiment was to determine the fold of the sheet during its handling during sorting. In the experiments, we considered different sizes of sorted fissures. In this paper, we present the results of the experiment for a sheet of $1000 \times 900 \mathrm{~mm}$ with a thickness of $1.6 \mathrm{~mm}$. We determined this dimension after consulting with Microstep, a.s.

In the course of the experiment, the suction cups were moved either diagonally (Fig. 3a) or vertically of the sheet (Fig. 3b).

The equipment needed to conduct the experiment: robot SEF VRS1D, Laser Tracker AT 901 and T- Scan, Suction cup type VAS 50 diameter $50 \mathrm{~mm}$ FESTO, gripper head, controller and PC.

If only the load in the transverse direction acts on the board and the board points can freely move in the $\mathrm{x}$ and $\mathrm{y}$ directions, then the relative deformations of the Kirchhoff model are:

$$
\begin{gathered}
\varepsilon_{x}=-z \frac{\partial^{2} w_{0}}{\partial x^{2}} \\
\varepsilon_{y}=-z \frac{\partial^{2} w_{0}}{\partial y^{2}} \\
y_{x y}=-2 z \frac{\partial^{2} w_{0}}{\partial x \partial y}
\end{gathered}
$$

For a certain value of the variable from $(-h / 2 \leq$ $z \leq h / 2)$, the deformation components of the plane (but now with $\varepsilon_{z}=0$ ), which, as seen from (1), linearly changes with the thickness of the sheet. From the 


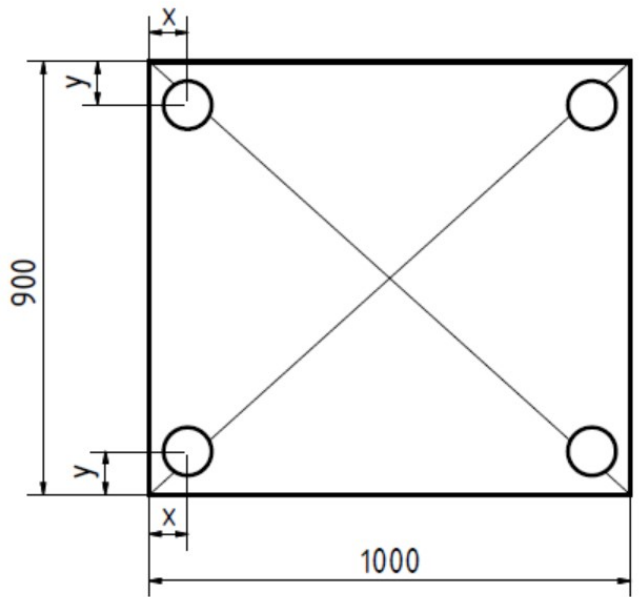

(A).

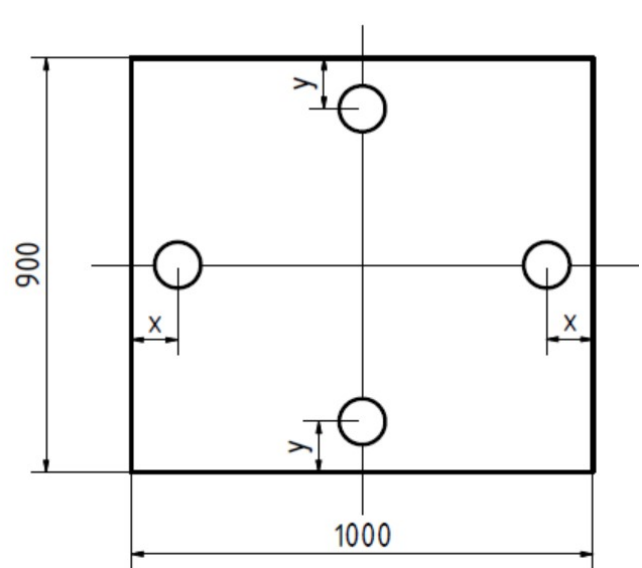

(B).

FiguRE 3. Grab handle of the sorting - sorting system.

physical (constitutive) equations valid for plane stress and isotropic material, we get the stresses in the sheet

$$
\begin{gathered}
\sigma_{x}=\frac{E}{1-\mu^{2}}\left(\varepsilon_{x}+\mu \varepsilon_{y}\right)= \\
=-\frac{E_{z}}{1-\mu^{2}}\left(\frac{\partial^{2} w_{0}}{\partial x^{2}}+\mu \frac{\partial^{2} w_{0}}{\partial y^{2}}\right) \\
\sigma_{y}=\frac{E}{1-\mu^{2}}\left(\varepsilon_{y}+\mu \varepsilon_{x}\right)= \\
=-\frac{E_{z}}{1-\mu^{2}}\left(\frac{\partial^{2} w_{0}}{\partial y^{2}}+\mu \frac{\partial^{2} w_{0}}{\partial x^{2}}\right) \\
\tau_{x y}=G_{y_{x y}}=\frac{E}{2(1+\mu)} y_{x y}= \\
=-\frac{E_{z}}{1-\mu^{2}}(1-\mu) \frac{\partial^{2} w_{0}}{\partial x \partial y}
\end{gathered}
$$

where $E$ is the modulus of the tensile/pressure elasticity, $\mu$ Poison number and $G$ modulus of material shear elasticity. The deflection area is also a neutral surface of tension, there are zero voltages on it and the sign changes. Extreme stresses are, as with bent beams, located on the bottom and top surfaces of the board.

The results of the internal forces are determined by the integration of tensions across the transverse direction unit width and are, therefore, often referred to in lower case letters:

$$
\begin{aligned}
m_{x}=\int_{-h / 2}^{h / 2}\left(\sigma_{x} d_{z}\right) z & = \\
=-\frac{E}{1-\mu^{2}}\left(\frac{\partial^{2} w_{0}}{\partial x^{2}}\right. & \left.+\frac{\partial^{2} w_{0}}{\partial y^{2}}\right) \int_{-h / 2}^{h / 2} z^{2} d z= \\
& =-D\left(\frac{\partial^{2} w_{0}}{\partial x^{2}}+\frac{\partial^{2} w_{0}}{\partial y^{2}}\right)
\end{aligned}
$$

$$
\begin{gathered}
m_{y}=\int_{-h / 2}^{h / 2}\left(\sigma_{y} d_{z}\right) z=-D\left(\frac{\partial^{2} w_{0}}{\partial y^{2}}+\frac{\partial^{2} w_{0}}{\partial x^{2}}\right) \\
m_{x y}=\int_{-h / 2}^{h / 2}\left(\tau_{x y} d_{z}\right) z=-D(1-\mu) \frac{\partial^{2} w_{0}}{\partial x \partial y}
\end{gathered}
$$

Coefficient D expresses the stiffness of the board:

$$
D=\frac{E}{1-\mu^{2}} \int_{-h / 2}^{h / 2} z^{2} d z=\frac{E h^{3}}{12\left(1-\mu^{2}\right)}
$$

\section{RESULtS}

Multiple sheets were used in the experiments as samples. In this paper, we present the results for a sheet metal of $1000 \times 900 \times 1.6 \mathrm{~mm}$. Below are the results of the experiment for different distributions of vacuum suction cups.;

\section{Vertical layout:}

Statistical data were used to correct the simulation and evaluation of the experimental results, where the mean, its minimum and the maximum was calculated from the measured data. The range was calculated from the minimum and maximum. The derivation was obtained by the second root of the scattering.

From these obtained values, the resulting correction coefficients, which are used to correlate the results from FEM, were then determined to approximate the real measured values. These correction coefficients can be used in the design of gripping heads, for which the maximum requirement is that, once the material is gripped, the manipulated object is subject to the smallest deflection. 10]

\section{Conclusion}

An Eexperimental observation of the change of a freeend deflection of the blank from the placement of the suction cups with its gravity load by using the laser $3 \mathrm{D}$ scanner was tested carried out for the method of detecting the deflection of the MKP. 


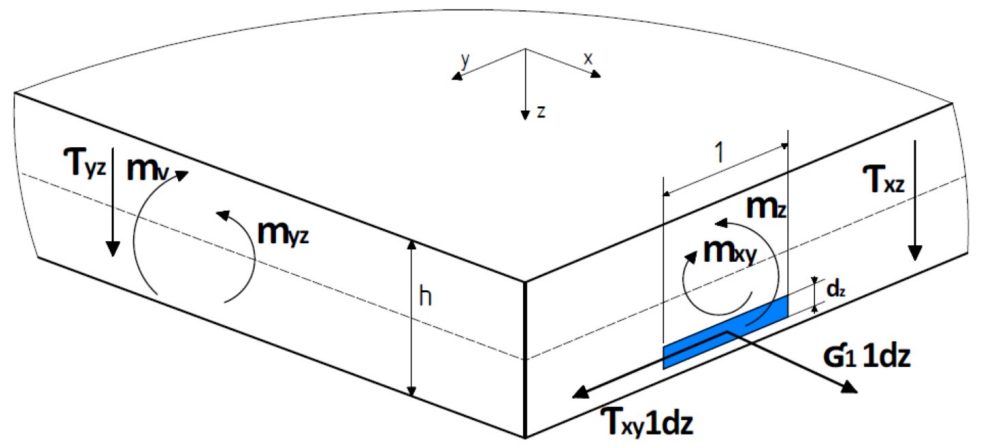

FiguRE 4. The resultant internal forces.
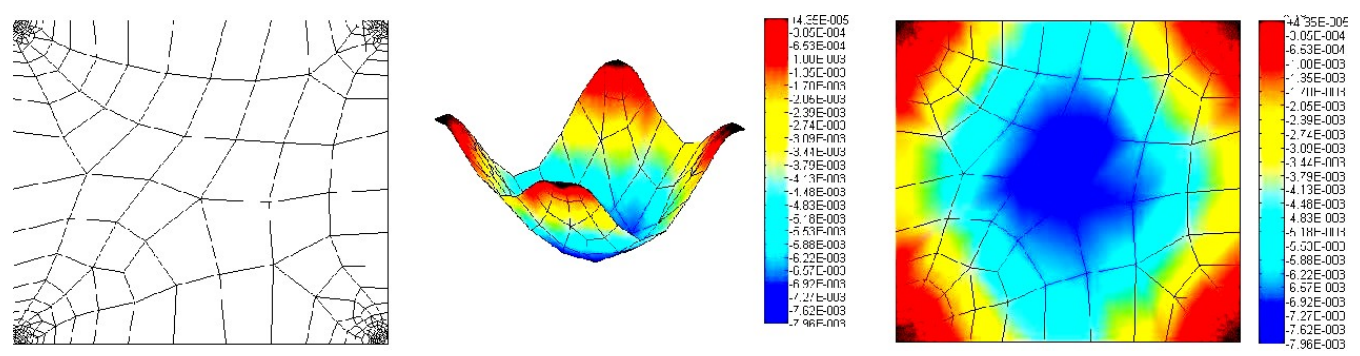

Figure 5. Position 1: a) mesh b) the detail of the deflection c) the resulting deflection $=7.96 \mathrm{~mm}$.
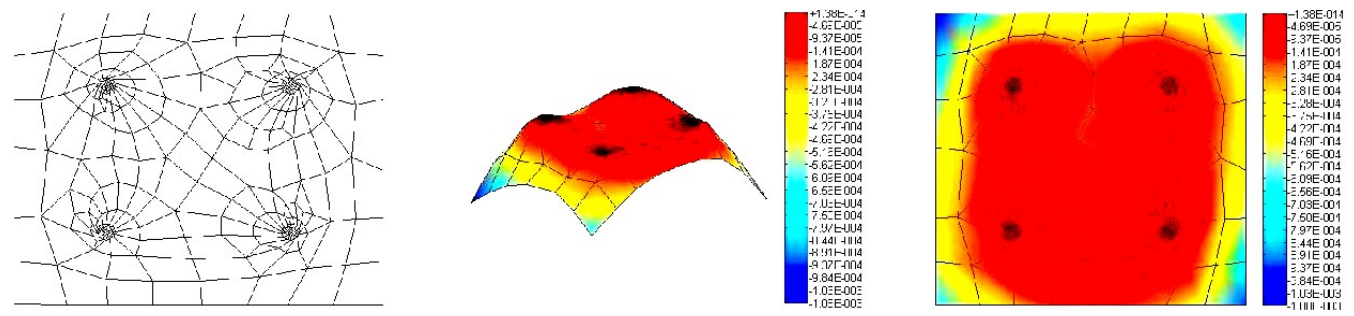

Figure 6. Position 4: The resulting deflection $=1.08 \mathrm{~mm}$.
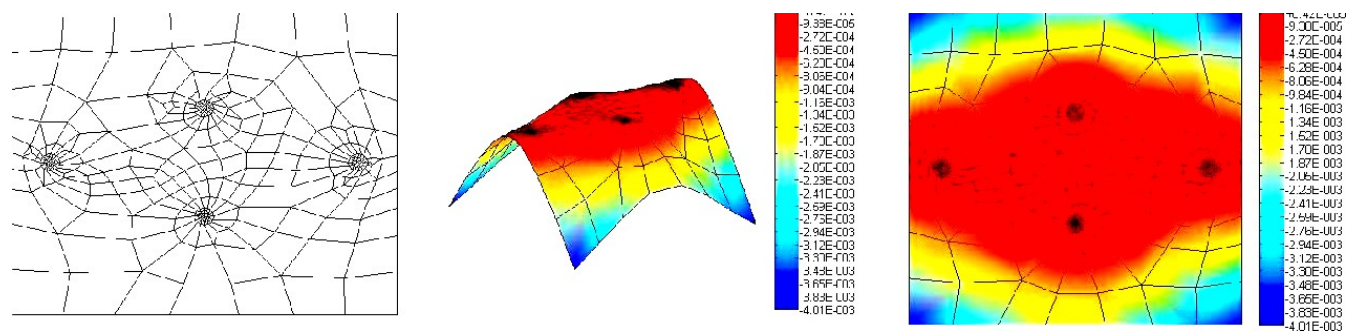

Figure 7. Position 1: The resulting deflection $=4.01 \mathrm{~mm}$.
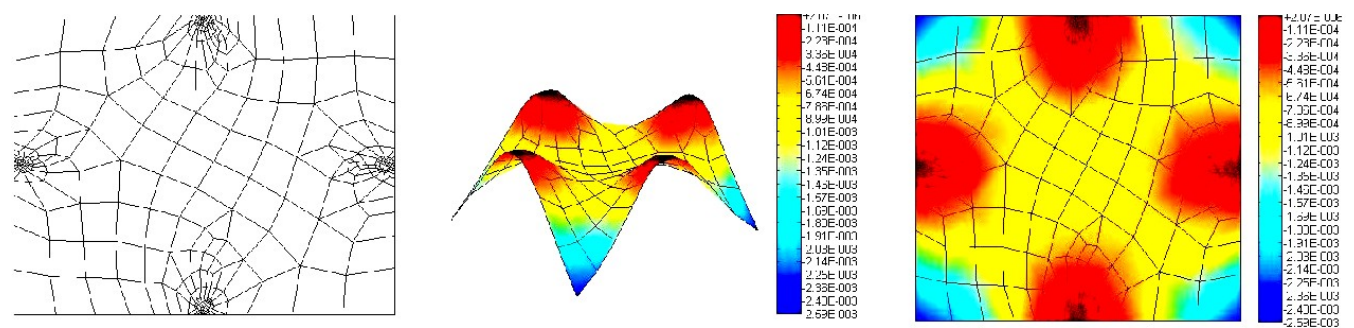

Figure 8. Position 4: The resulting deflection $=2.59 \mathrm{~mm}$. 


\begin{tabular}{cccc|ccc}
\hline \multirow{2}{*}{ No } & \multicolumn{2}{c}{ DIAGONAL LAYOUT } & \multicolumn{3}{c}{ VERTICAL LAYOUT } \\
\cline { 2 - 6 } & Polyworks & Matlab & Solidworks & Polyworks & Matlab & Solidworks \\
\hline 1 & 15.569 & 7.96 & 9.44 & 14.532 & 4.01 & 6.448 \\
2 & 7.598 & 2.65 & 3.025 & 15.08 & 3.11 & 4.23 \\
3 & 8.428 & 0.894 & 0.736 & 13.31 & 3.56 & 3.641 \\
4 & 7.574 & 1.08 & 1.06 & 8.77 & 2.59 & 2.872 \\
5 & 13.24 & 3.45 & 4.18 & & & \\
6 & 20.028 & 8.51 & 8.628 & & & \\
\hline
\end{tabular}

TABLE 1. Resulting deflections values.

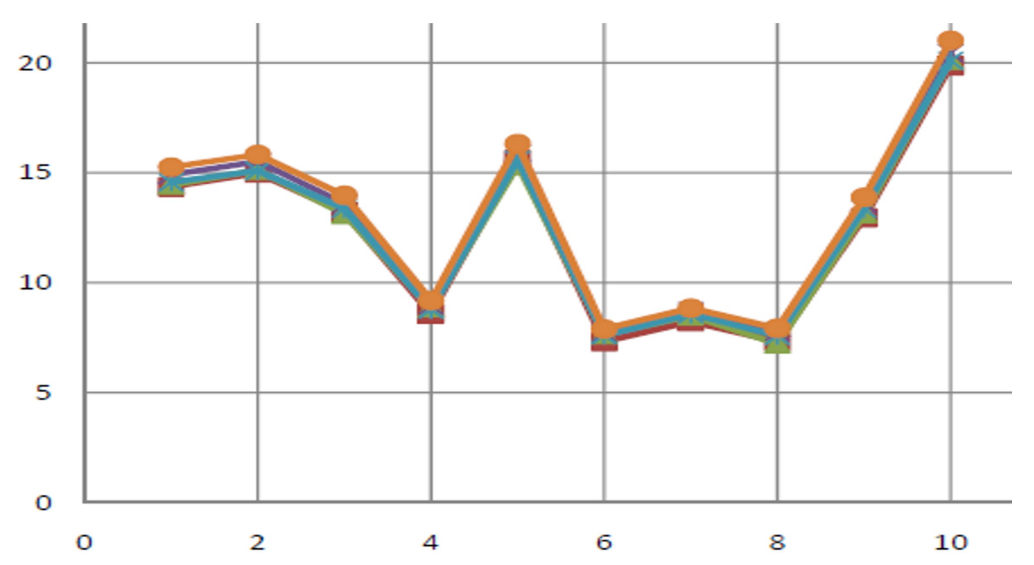

FigurE 9. Graphic representation of the deflection at a thickness of $1.6 \mathrm{~mm}$ using correction coefficients.

The experiment confirmed that the differences between the computational model and the results obtained from the simulation and experiment differed by a rather large value, which was not negligible for the given manipulation schemes.

The presented process of the proposal contributes to streamlining and simplifying the work of the designer in the design of vacuum undercuts. It helps the user to select the correct size and number of suction cups when handling steel sheets and then choose their optimal layout in the suction cup design. In the calculations, the dynamic aspect of the system load was not solved.

\section{ACKNOWLEDGEMENTS}

The research presented in this paper is an outcome of the project No. APVV-16-0485 "Tools for compaction of biomass cast from progressive refractory cast iron." funded by the Slovak Research and Development Agency.

\section{REFERENCES}

[1] A. Varga, A. Smelík, P. Varga. MSF - fiber laser cutting machines with bevel cutting. Strojárstvo 5, 2016.

[2] J. xiong Chen, S. wen Lin, X. long Zhou, Y. liu Tu. An on-machine error calibration method for a laser micromachining tool. Precision Engineering 47:239 248, 2017. DOI:10.1016/j.precisioneng.2016.08.009

[3] A. Marwan, M. Simic, F. Imad. Calibration method for articulated industrial robots. Procedia Computer Science 112:1601 - 1610, 2017. Knowledge-Based and Intelligent Information \& Engineering Systems: Proceedings of the 21st International Conference, KES-20176-8 September 2017, Marseille, France, DOI:10.1016/j.procs.2017.08.246.
[4] H. Castro, M. Burdekin. Dynamic calibration of the positioning accuracy of machine tools and coordinate measuring machines using a laser interferometer. International Journal of Machine Tools and Manufacture 43(9):947 - 954, 2003. DOI:10.1016/S0890-6955(03)00083-X

[5] J. Slamka, M. Jedinák, M. Tolnay, et al. Automatic manipulation of parts made from yielding material. In OPTIROB 2013, vol. 332 of Applied Mechanics and Materials, pp. 423-430. Trans Tech Publications Ltd, 2013. DOI:10.4028/www.scientific.net/AMM.332.423.

[6] A. Gürsel, S. Bijan. A systematic technique to estimate positioning errors for robot accuracy improvement using laser interferometry based sensing. Mechanism and Machine Theory 40(8):879 - 906, 2005. DOI:10.1016/j.mechmachtheory.2004.12.012

[7] G. T. Smith. Machine Tool Metrology. Springer, Cham, Switzerland, 2016. DOI:10.1007/978-3-319-25109-7

[8] L. Sobotová, R. Králiková, M. Badida. The analysis of chosen material properties at thermal drilling. In Material Engineering Practice IX, vol. 635 of Key Engineering Materials, pp. 35-40. Trans Tech Publications Ltd, 2015. DOI:10.4028/www.scientific.net/KEM.635.35.

[9] L. Sobotová, M. Badida. The new knowledge of environmentally friendly joints made by thermal drilling. In Surface Engineering and Materials in Mechanical Engineering, vol. 818 of Materials Science Forum, pp. 272-275. Trans Tech Publications Ltd, 2015. DOI:10.4028/www.scientific.net/MSF.818.272.

[10] J. Slamka. Automatic handling of parts made of flexible materials. Ph.D. thesis, Slovak University of 
Technology in Bratislava, Faculty of Mechanical Engineering, 2012.

[11] M. Paweł, P. Bartosz. Rapid method to determine accuracy and repeatability of positioning of numerically controlled axes. International Journal of Machine Tools and Manufacture 137:1 - 12, 2019.

DOI:10.1016/j.ijmachtools.2018.09.006 